\title{
Modelling the Wake Flow Behind a Model of Horizontal Axis Wind Turbine using RANS Approach: A Comparative Study
}

\author{
Prof. Dr. Abd Alnaby Kabeel, Prof. Dr. El-Sayed El-Aguoz, Prof. Dr. Ayman I. Bakry \\ and Mohammad R. Shahin \\ Mechanical Power Department, Faculty of Engineering, Tanta University, Egypt. \\ Corresponding author email: M.Raafat@f-eng.tanta.edu.eg
}

\begin{abstract}
Steady CFD simulations, which is based on Reynolds Average Navier-Stokes (RANS) approach, were conducted for a model of Horizontal Axis Wind Turbine (HAWT) and compared with experimental data in order to assess the accuracy of these models in simulating the wake flow of HAWTs. The models can be categorized into two categories: fully developed models (Standard $k-\varepsilon$ model, Realizable $k-\varepsilon$ model, and SST $k-\omega$ model) and

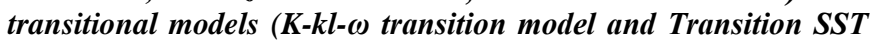
Model). In this paper, ANSYS FLUENT 19.2 was being used to execute the simulations of the model turbine standing on a closedloop wind tunnel at Norwegian University of Sciences and Technology (NTNU). Experiments were operating on a high turbulence intensity uniform inflow. Velocity, turbulence intensity, and turbulent kinetic energy profiles are illustrated at two downstream cross sections. Furthermore, contours of these parameters are set to investigate the developing of wake flow behind the turbine. It is demonstrated that models reasonably predict the velocity profile at the wake region. Transitional models are more accurate in predicting the power of the turbine. Turbulent kinetic energy and turbulence intensity were underestimated for all models.
\end{abstract}

Keywords- CFD, Turbulence Modelling, RANS, Wake Effect, HAWT.

\section{INTRODUCTION}

According to Global Wind Report [1] , about $60 \mathrm{GW}$ from wind farms have been installed during 2019, resulting in the rise of cumulative capacity to $650 \mathrm{GW}$ around the world. Such increasing deployment of wind farms requires explicit analysis of the physical aspects of wind turbines and their interaction within wind farms to reduce the sources of power losses. Wake effect is considered one of the highest disruptions of wind energy evolution with an estimated power loss of $10-20 \%$ in the wind farm [2].

Wake is the region in which velocity is decreased and turbulence intensity is increased due to momentum extraction by the upstream turbines entailing power losses for the following downstream turbines. Moreover, downstream turbines experience high loads because of overlapping between wakes from different turbines, thereby its longevity is significantly reduced []ㅡ.

On a larger scale, Wake effect exceeds its impacts over the farm to downstream farms. In reference [4], authors have illuminated the physical, economical, and legal sides of wake effect. They demonstrated that the wake effect of an upstream farm can extend for $50 \mathrm{~km}$, resulting in a power loss of nearly $5 \%$ for the downstream farm. This requires a legal move to organize the installation of new farms or any construction projects upstream the operating farms.

\section{LITERATURE REVIEW}

Complexity of wake flow arises from the fact that it is time dependent, three-dimensional, and a fluctuating flow with a wide scale of eddies. Experimental and large eddy simulation (LES) studies are the most trusted approaches to highlight the physics and study the influence of different parameters in wind farms, but both are time consuming and require high finance. On the other hand, analytical models, with low required computing resources, have already been used to optimize farm layouts. Nevertheless, when it is compared with experimental and CFD studies, it shows inadequacies in representing wake behavior and lose the advantage as it cannot help in cases such as different atmospheric conditions, meandering flow, and unsteadiness capturing []ㅡ.

a LES simulation [] ] was conducted and validated by NREL phase VI wind experiments showing high accuracy and demonstrated the shortcomings of the analytical models like Larsen, Jensen, and Frandsen. it was found that precision differs from analytical model to another, but generally they show less accuracy in describing velocity field, overprediction of wake expansion and low precision of recovery rate. Furthermore, these models cannot visualize wake behavior such as separation, mixing mechanism, deflection, wake formation, and fading of vortices.

Also, reference [7] studied the wake characteristics of the two-bladed NREL phase VI wind turbine using Large eddy simulation at a uniform upstream velocity and low turbulence intensity. The rotor was fully represented, while nacelle and tower were simplified to lower the computational cost. The study showed an accurate result compared to the experimental data, it managed to perfectly validate the simulation chordwise pressure coefficients with the experimental values guarantee high accuracy prediction of aerodynamic behavior of the turbine, then wake is thought to be simulated accurately. The study defines the boundary between near wake and far wake regions. The former is the direct region behind the turbine and extends to nearby 5 times the turbine diameter, it is 
characterized by a decreased velocity and high turbulence level. The latter, far wake region, is the region at which velocity is recovered partially and vortices are damped.

For the last view years, an increasing research studies based on large eddy simulation have been examined showing the superior of LES technique in predicting and investigating physics details such as separation on the blade, deflection of the wake at yawed turbines [8], vortices formation and their coherent structure, and last but not least estimating the wake flow at different atmospheric boundary conditions [9]. Even though the evolution of the computational resources, LES technique still unaffordable and requires more development in hardware and software capabilities[7].

RANS approach has the advantage of acceptable results for a wide range of applications with relatively lower time and computing costs. $\mathrm{k}-\varepsilon$ model and SST $\mathrm{k}-\omega$ model are considered the familiar models of this approach. For k- $\varepsilon$ model, A general conclusion of insufficient results is found in the literature for the application of wind turbines $[10,11]$, the flaw had been well demonstrated in the underprediction of velocity deficit in the near wake region and misrepresentation of turbulence intensity. The reasons were returned to isotropic assumption and excess estimation of turbulent diffusion that results in high dissipation at the region behind the turbine. Many modifications had been made to enhance the model performance such as references [12-14]. Although, it countered some numerical issues and requires more enhancements.

Regarding SST k- $\omega$ model, it shows acceptable agreements with experimental data for moderate and high inflow velocities, otherwise lower accuracy for low inflow velocities by reference to [15]. Evaluating this model in different boundary conditions and yaw still needs more evaluation.

In this study, predictions of fully turbulent models (Standard k- $\varepsilon$ model, Realizable k- $\varepsilon$ model and SST k- $\omega$ model) and transitional models (K-kl- $\omega$ transition model and Transition SST Model) are validated by Norwegian University of Sciences \& Technology (NTNU) experiments [16]. The majority of CFD studies in literature, which simulate wind tunnel experiments, adopt fully turbulent models neglecting the fact that Reynolds number in wind tunnels is approximately in the transitional flow regime. In this paper, transitional models were selected as the Reynolds number based on the blade tip cross section is $10^{5}$, thereby the flow is a transitional flow.

Results show that most of models predicted power of the turbine reasonably with slight deviations. For the near region behind the turbine, all models show overestimation of velocity deficit. Fully turbulent models show accurate prediction of velocity profile at the far region. Transitional models underestimated velocity deficit. Shortages of models appear obviously in turbulence intensity and turbulent kinetic energy estimation. Understanding deficiencies of these models can help in reformulation of their terms for better performance.

\section{GOVERning EQUATIONS AND TURBULENCE MODELLING}

\subsection{Governing Equations:}

In the present research, the Averaged Navier Stokes equations (RANS) are selected to model the flow. Averaged equations are formulated by replacing the instantaneous velocity in exact Navier Stokes equations by the sum of mean velocity $U$ and fluctuating velocity yielding (1) and (2) in Einstein notation [17] considering the steady assumption.

$\frac{\partial}{\partial x_{i}}\left(\rho u_{i}\right)=0$

$\frac{\partial}{\partial x_{i}}\left(\rho u_{i} u_{j}\right)=-\frac{\partial p}{\partial x_{i}}+\frac{\partial}{\partial x_{j}}\left[\mu\left(\frac{\partial u_{i}}{\partial x_{i}}+\frac{\partial u_{j}}{\partial x_{j}}+\right.\right.$
$\left.\left.\frac{2}{3} \delta_{i j} \frac{\partial u_{l}}{\partial x_{l}}\right)\right]+\frac{\partial}{\partial x_{j}}\left(-\rho \overline{u_{l}^{\prime} u_{j}^{\prime}}\right)$

Reynolds stresses $\overline{u_{\imath}^{\prime} u_{\jmath}^{\prime}}$ represents the influence of turbulence on the mean flow. Two approaches were evolved to model Reynolds stresses: eddy viscosity models and Reynolds-stress models (RSM) [18]. We used in our research standard k- $\epsilon$, realizable k- $\epsilon$, SST k- $\omega, \mathrm{K}-\mathrm{kl}-\omega$ transition and Transition SST Models which all are based on the first approach. Eddy viscosity models is based on Boussinesq hypothesis assuming that turbulence leads to additional viscosity effect resembling molecular viscosity effect i.e. Reynolds stresses has a correlation with the mean rate of deformation in the flow as in equations (3) and (4).

$$
\begin{aligned}
& -\rho \overline{u_{\imath}^{\prime} u_{\jmath}^{\prime}}=2 \mu_{t} S_{i j}-\frac{2}{3} \rho k \delta_{i j} \\
& S_{i j}=\frac{1}{2}\left[\frac{\partial u_{i}}{\partial x_{j}}+\frac{\partial u_{j}}{\partial x_{i}}\right]
\end{aligned}
$$

Where $\mu_{t}$ is eddy viscosity, which is needed to be modeled, $S_{i j}$ is the mean strain rate tensor, $\rho$ is the density, $\mathrm{k}$ is the turbulent kinetic energy, and $\delta_{i j}$ is the Kronecker delta function.

\subsection{Standard k- $\varepsilon$ model}

Additional two equations are formulated to model the turbulent viscosity that was identified by Boussinesq. The two equations represent the production and destruction of turbulent kinetic energy.

$$
\begin{aligned}
& \mu_{t}=\rho C_{\mu} \frac{k^{2}}{\varepsilon} \\
& \frac{\partial}{\partial x_{i}}\left(\rho k u_{i}\right)=\frac{\partial}{\partial x_{j}}\left[\left(\mu+\frac{\mu_{t}}{\sigma_{k}}\right) \frac{\partial k}{\partial x_{j}}\right]+G_{k}+G_{b}-\rho \varepsilon \\
& -Y_{M}+S_{k}
\end{aligned}
$$




$$
\begin{array}{r}
\frac{\partial}{\partial x_{i}}\left(\rho \varepsilon u_{i}\right)=\frac{\partial}{\partial x_{j}}\left[\left(\mu+\frac{\mu_{t}}{\sigma_{\varepsilon}}\right) \frac{\partial \varepsilon}{\partial x_{j}}\right]+C_{1 \varepsilon} \frac{\varepsilon}{k}\left(G_{k}\right. \\
\left.+C_{3 \varepsilon} G_{b}\right)-C_{2 \varepsilon} \rho \frac{\varepsilon^{2}}{k}+S_{\varepsilon}
\end{array}
$$

Where $\mu$ is the molecular viscosity, $G_{k}$ represents the generation of turbulence kinetic energy due to the mean velocity gradients, $G_{b}$ is the generation of turbulence kinetic energy due to buoyancy, $Y_{M}$ represents the contribution of the fluctuating dilatation in compressible turbulence to the overall dissipation rate. $C_{1 \varepsilon}, C_{2 \varepsilon}, C_{3 \varepsilon}$ and $C_{\mu}$ are constants. $\sigma_{k}$ and $\sigma_{\varepsilon}$ are the turbulent Prandtl numbers for $\mathrm{M}$ and $\mathrm{N}$, respectively. $S_{k}$ and $S_{\varepsilon}$ are user-defined source terms. And $C_{1 \varepsilon}=1.44, C_{2 \varepsilon}=1.92, C_{\mu}=0.09, \sigma_{k}=1.0, \sigma_{\varepsilon}=1.3$

For the cases of low Reynolds number such as near wall boundary, equations (5), (6), and (7) are multiplied by damping functions which guarantee that viscous stresses are dominant over Reynolds stresses. But it showed unreliability in a variety of applications.

\subsection{Realizable k- $\varepsilon$ model}

To overcome shortages of standard model, Improved equation for the dissipation rate was derived, moreover, $C_{\mu}$ is formulated to be variable instead of constant value to avoid mathematical non-realizability for the cases of high mean strain rate. [19] proposed a reasonably model which makes $C_{\mu}$ sensible for the rate of deformation, turbulence production $(\mathrm{k})$, and turbulence dissipation $(\varepsilon)$. For more detailed equations, it is advised to be familiarized with [17] and [19].

\subsection{Standard k- $\omega$ model}

Wilcox (standard) $\mathrm{k}-\omega$ model replaces the dissipation rate in $\mathrm{k}-\varepsilon$ model with turbulence frequency $\left(\omega=\mathrm{k} / \varepsilon\left[\mathrm{s}^{-1}\right]\right)$, thereby eddy viscosity be in the form of equation (8). Reynolds stresses are calculated as in $\mathrm{k}-\varepsilon$ model, where $\mathrm{k}$ and $\omega$ are modeled by (9) and (10).

$$
\begin{aligned}
& \mu_{t}=\rho \alpha^{*} \frac{k}{\omega} \\
& \frac{\partial}{\partial x_{i}}\left(\rho k u_{i}\right)=\frac{\partial}{\partial x_{j}}\left[\left(\mu+\sigma_{k} \mu_{t}\right) \frac{\partial k}{\partial x_{j}}\right]+G_{k}-Y_{k}+S_{k} \\
& \frac{\partial}{\partial x_{i}}\left(\rho \omega u_{i}\right)=\frac{\partial}{\partial x_{j}}\left[\left(\mu+\sigma_{\omega} \mu_{t}\right) \frac{\partial \omega}{\partial x_{j}}\right]+G_{\omega}-Y_{\omega}+S_{\omega}
\end{aligned}
$$

Where $G, Y$, and $S$ are production, dissipation, and source terms, respectively. Eddy viscosity $\mu_{t}$ is multiplied by the coefficient $\alpha^{*}$ to correct its value at low Reynolds numbers.

The Wilcox k- $\omega$ model performs well for boundary layer flows with adverse pressure gradients but have difficulties in the case of external flow because of its dependency on the value of freestream turbulence frequency $\omega$ at the inlet boundary [20].

\subsection{SST k- $\omega$ model}

A smooth transition between Standard k- $\varepsilon$ model and Wilcox $\mathrm{k}-\omega$ model is achieved by blending functions which activate the k- $\omega$ model near the wall region and $k-\varepsilon$ in the far field for better performance.

Through [20-23], Menter had suggested and developed the SST k-w model preserving the Reynolds stresses and transport equation of $\mathrm{k}$, the same as Wilcox $\mathrm{k}-\omega$ model, but the $\varepsilon$-equation is modified.

$$
\frac{\partial}{\partial x_{j}}\left(\rho \omega u_{j}\right)=\frac{\partial}{\partial x_{j}}\left[\begin{array}{c}
\left.\left(\mu+\sigma_{\omega} \mu_{t}\right) \frac{\partial \omega}{\partial x_{j}}\right]+G_{\omega}-Y_{\omega}+D_{\omega} \\
+S_{\omega}
\end{array}\right.
$$

Where $G, Y, D$, and $S$ are production, dissipation, the cross diffusion, and source terms, respectively. A detailed formulation for all terms is represented at [17]. It is aforementioned that the strain rate (S) contains an important function named the blending function $F_{1}$. Its value determines whether $k-\omega$ is switched on or k- $\epsilon$ model. Eddy viscosity is limited in (12) To prevent the excessive turbulence production at stagnation conditions by a limiter $F_{2}$.

$$
\begin{aligned}
& \mu_{t}=\rho \frac{k}{\omega} * \frac{1}{\left[\frac{1}{\alpha^{*}}, \frac{S F_{2}}{a_{1} \omega}\right]} \\
& S=f\left(F_{1}\right)
\end{aligned}
$$

\subsection{Transition SST model}

Modifications were applied for the SST k- $\omega$ model spreading its applicability to laminar, transitional, and fully turbulent flows. Transitional SST model is a Four transport equations model (k, $\left.\omega, \gamma, R e_{\theta, t}\right)$. $\mathrm{k}$ and $\omega$ (14) and (15) have the same formula as the SST k- $\omega$ model, but three terms are modified depending on the flow regime. These terms are production, dissipation, and the blending function $F_{1}$.

$$
\begin{gathered}
\frac{\partial}{\partial x_{i}}\left(\rho k u_{i}\right)=\frac{\partial}{\partial x_{j}}\left[\left(\mu+\sigma_{k} \mu_{t}\right) \frac{\partial k}{\partial x_{j}}\right]+\gamma G_{k}-Y_{k}^{*}+S_{k} \\
\frac{\partial}{\partial x_{i}}\left(\rho \omega u_{i}\right)=\frac{\partial}{\partial x_{j}}\left[\begin{array}{c}
\left.\left(\mu+\sigma_{\omega} \mu_{t}\right) \frac{\partial \omega}{\partial x_{j}}\right]+G_{\omega}-Y_{\omega}^{*}+D_{\omega} \\
+S_{\omega}
\end{array}\right.
\end{gathered}
$$

Where $\gamma$ is the turbulence intermittency? For laminar flow $\gamma=$ 0 ; so, the production term equals zero. For $\gamma=1$, The production term is the same as the SST k- $\omega$ model i.e. fully turbulent model. Finally, if $0<\gamma<1$, then it is transitional. $Y_{k}^{*}$ is the modified dissipation term in which a limiter is used to damp and dissipate any fluctuations on the freestream. blending function $F_{1}$ is modified for correct switching between $\mathrm{k}-\omega$ and $\mathrm{k}-\varepsilon$ models.

The two additional transport equations $\gamma$ and $R e_{\theta, t}$ are full of empirical and experimental equations to be closed. $\gamma$ is for 
the intermittency and $R e_{\theta, t}$ for the transition onset criteria, in terms of momentum-thickness Reynolds number.

\subsection{K-kl- $\omega$ transition model}

A three-transport equations model has been represented by Walters and Cokljat [24]. The model is based on the $\mathrm{k}-\omega$ model, modifying the two transport equations $\mathrm{k}$ and $\omega$. The third transport equation is the laminar kinetic energy $\left(k_{L}\right)$ equation, which has been formulated to predict the small fluctuations in low Reynolds number flow region before the transitional boundary layer. Authors demonstrated that the equations are based on physical approach decreasing the need of empirical equations.

$$
\begin{aligned}
\frac{\partial}{\partial x_{i}}\left(\rho k_{T} u_{i}\right)=\frac{\partial}{\partial x_{j}} & {\left[\left(v+\frac{\sigma_{T}}{\sigma_{k}}\right) \frac{\partial k_{T}}{\partial x_{j}}\right]+\mathrm{P}_{\mathrm{K}_{\mathrm{T}}}+\mathrm{R} } \\
& +\mathrm{R}_{\mathrm{NAT}}-\omega \mathrm{k}_{\mathrm{T}}-D_{T} \\
\frac{\partial}{\partial x_{i}}\left(\rho k_{L} u_{i}\right)=\frac{\partial}{\partial x_{j}}\left[v \frac{\partial k_{L}}{\partial x_{j}}\right]+\mathrm{P}_{\mathrm{K}_{\mathrm{L}}}-\mathrm{R}-\mathrm{R}_{\mathrm{NAT}} & -D_{L} \\
\frac{\partial}{\partial x_{i}}\left(\rho \omega u_{i}\right)=\frac{\partial}{\partial x_{j}}[ & \left.\left(v+\frac{\sigma_{T}}{\sigma_{\omega}}\right) \frac{\partial \omega}{\partial x_{j}}\right]+\mathrm{C}_{\omega 1} \frac{\omega}{k_{T}} P_{k_{T}} \\
& +\left(\frac{\mathrm{C}_{\omega \mathrm{R}}}{f_{w}}-1\right) \frac{\omega}{k_{T}}\left(\mathrm{R}+\mathrm{R}_{\mathrm{NAT}}\right) \\
& -\mathrm{C}_{\omega 2} \omega^{2} \\
& +\mathrm{C}_{\omega 3} f_{\omega} \alpha_{T} f_{w}^{2} \frac{\sqrt{k_{T}}}{d^{3}}
\end{aligned}
$$

\section{EXPERIMENTAL SETUP}

\subsection{Wind tunnel}

Wind tunnel experiments were carried out at the Norwegian University of Science and Technology (NTNU). A closed-loop wind tunnel was established at NTNU with $2.7 \mathrm{~m}$ cross section, $1.81 \mathrm{~m}$ height and $11.15 \mathrm{~m}$ long. It is driven by $220 \mathrm{~kW}$ fan positioned at the downstream side.

\subsection{Operating conditions}

A uniform velocity profile of $10 \mathrm{~m} / \mathrm{s}$ was established. Turbulence was generated by a uniform grid, made from wooden bars, at the entrance of the tunnel. Turbulence intensity decays downstream until reaches a value of 0.10 at the position of the turbine. The model wind turbine was set at a zero angle for both pitch and yaw angles.

Velocity data was being logged using a two component Laser doppler anemometer (LDA). The axial and the vertical velocity components were recorded by the LDA.

Power of the turbine equals the multiplication of rotational speed by the torque. Radial speed was measured by an optical (rpm) sensor, where torque was measured by torque transducer. Thrust force was measured using a six-component force balance.

\section{Simulation SETUP}

\subsection{Domain and Meshing}

The computational domain shown in Figure 1 has the same geometry of the closed-loop wind tunnel. To accomplish the steady state for the rotating turbine, Multiple Reference Frame (MRF) approach was adopted. For MRF approach, two separate zones are defined: stationary zone for the tunnel and a rotating zone enclosing the turbine rotor and the hub. Additionally, equations are modified by considering a frame

of reference, that rotates with the blades, preventing cells to rotate. Therefore, we obtain two frames: the first is the stationary frame and the second is the moving frame that rotate with the blades.

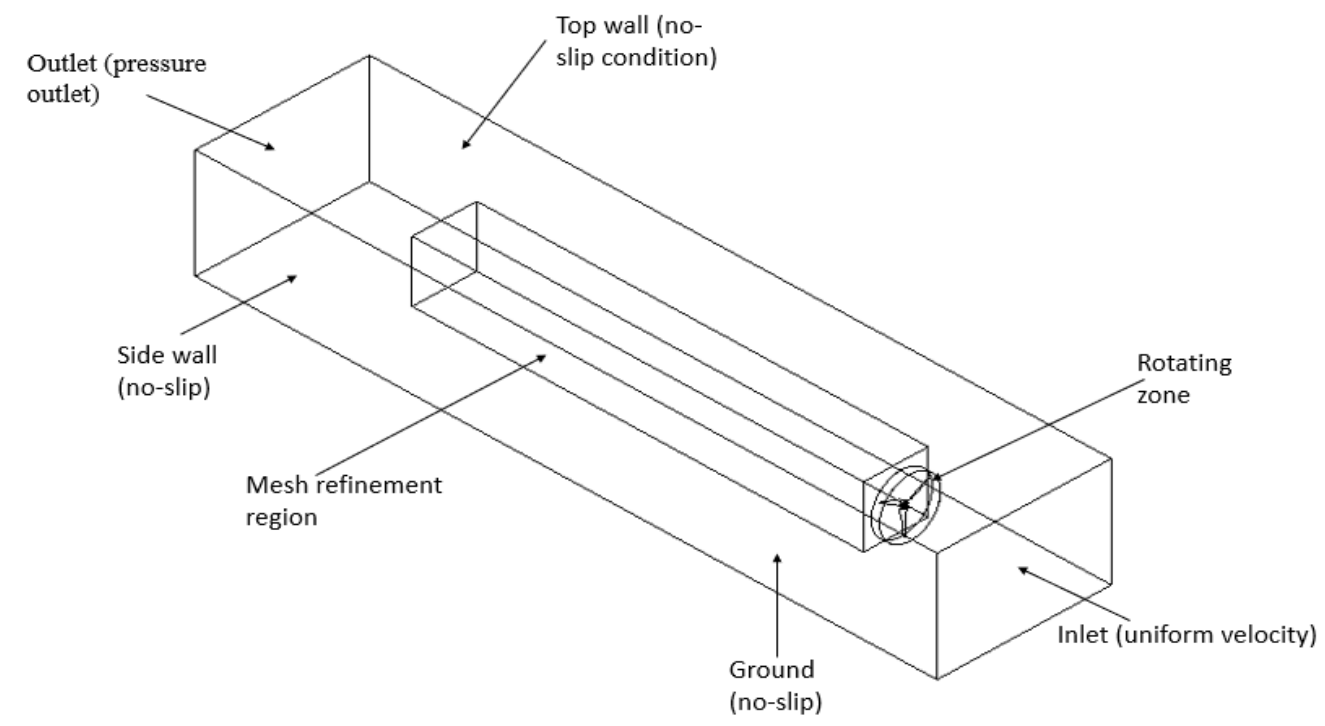

Figure 1 . The computational domain and the corresponding boundary conditions. 
Considering that the origin of the MRF is displaced by a vector $\overrightarrow{r_{o}}$ rotating by angular velocity $\vec{\omega}$, and translating with a linear velocity $\overrightarrow{v_{t}}$. Then, the domain is defined w.r.t the rotating frame in our case and for an arbitrary fluid element has a vector $\vec{r}$ relative to the MRF. Thus, the velocity becomes in the forms of equation (19).

$$
\overrightarrow{v_{r}}=\vec{v}-\left[\overrightarrow{v_{t}}-\vec{\omega} \times \vec{r}\right]
$$

Where $\overrightarrow{v_{r}}$ the relative velocity which is viewed from the MRF and $\vec{v}$ the absolute velocity (to the stationary frame).

To solve the equation of motion, we selected the absolute velocity formulation that provided by ANSYS; so, the equation of continuity and momentum are modified on the forms of equations (20) and (21), respectively.

$$
\begin{aligned}
& \nabla . \rho \overrightarrow{v_{r}}=0 \\
& \nabla \cdot\left(\rho \overrightarrow{v_{r}} \vec{v}\right)+\rho\left[\vec{\omega} \times\left(\vec{v}-\overrightarrow{v_{t}}\right)\right]=-\nabla P+\nabla \cdot \bar{\tau}+\vec{F}
\end{aligned}
$$

Where $\bar{\tau}$ is the viscous stress.

The domain was meshed with $7.67 \times 10^{6}$ unstructured tetrahedral cells. Finer mesh cells were adopted for the region surrounding the turbine and the downstream region where the wake is expected to expand. Blade surfaces were meshed with $2.4 \mathrm{~mm}$ cell size and the rotating zone with $10 \mathrm{~mm}$ cell size. Furthermore, the volume of the tunnel was meshed by $250 \mathrm{~mm}$ cell size. A refinement region was meshed as a rectangular prism, which has $1.6 \times 1.7 \mathrm{~m}^{2}$ dimensions, by $30 \mathrm{~mm}$ cell size. This refinement is selected to cover the region where wake is expected to exist.

A series of setups, in which mesh cell size had been altered for blade surfaces when other cells are constant and vice versa, were operated. It was found that finer blades cells, that increase cells count by $40 \%$, marginally increases power coefficient by $2 \%$. Otherwise, increasing wake region cells size slightly alters the velocity profile, but significant impact rises for the turbulence intensity and TKE maximum values. SST $\mathrm{k}-\omega$ turbulence model was selected for the mesh independence study.

\subsection{Numerical Setup}

Simulations were conducted by ANSYS Fluent 19.2. Pressure based solver was used with coupled scheme for pressure-velocity coupling. A second order upwind scheme was adopted for the momentum, turbulence kinetic energy, and the turbulence dissipation rate. Convergence criteria was set as 10-4 for continuity equation and 10-5 for the rest of equations. Reports for moment and thrust on the rotor were set to monitoring the convergence.

\subsection{Boundary Conditions}

Turbine rotor and walls of the tunnel were set as a no-slip boundary condition, and Inflow boundary was set as velocity inlet. Pressure outlet boundary condition is selected for the outlet.

Simulations for an empty tunnel was conducted to validate the model and the boundary conditions. Experimentally, a constant uniform velocity of $10 \mathrm{~m} / \mathrm{s}$ along the tunnel had been maintained, as the roof of the tunnel was adjusted for zero pressure gradient. From simulation, velocity profile Error! Reference source not found. at $\mathrm{x} / \mathrm{D}=0,3$, and 6 is represented showing low deviation of velocity magnitude with a max. error of nearly $3 \%$ at $x / D=6$. Where, Figure 3 shows that gauge pressure difference along the tunnel is less than $5 \mathrm{~Pa}$ which can be neglected.

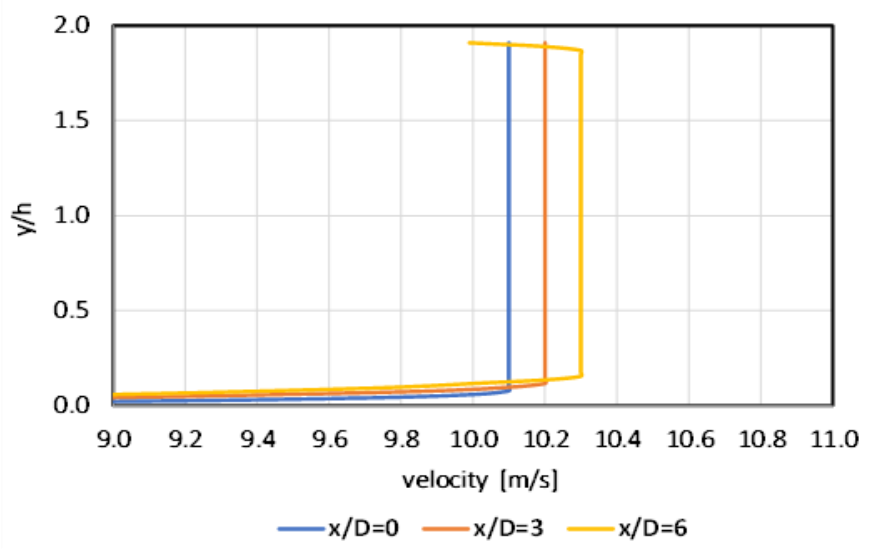

Figure 2.Velocity profile at different cross section at empty tunnel

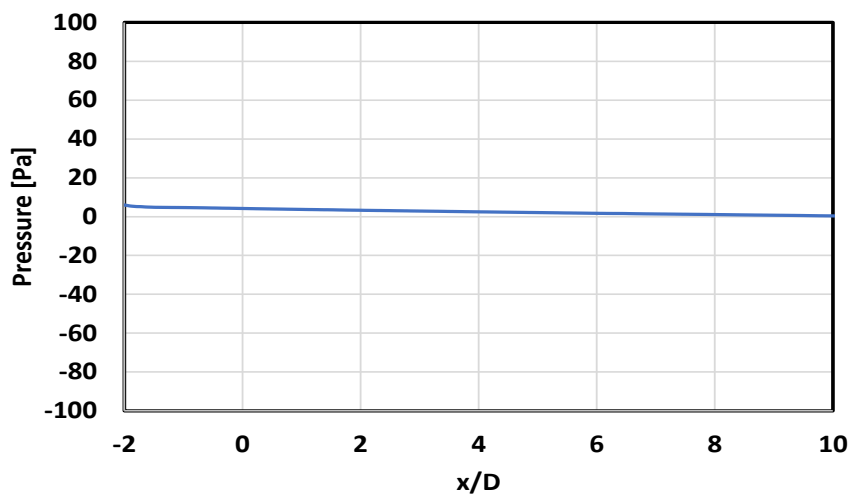

Figure 3. Static pressure at a line located on the top wall of the tunnel extending from the inlet to the outlet

During the rotating of the turbine, velocity profile at the position of the turbine achieves the experimental condition $(\mathrm{u}=10 \mathrm{~m} / \mathrm{s})$. Also, turbulence intensity was guaranteed to be 0.1 at the rotor position like the experimental setup by adjusting values of inlet TI and viscosity ratio (VR). 


\subsection{The model turbine}

The turbine is located at $1.8 \mathrm{~m}$ far from the inlet. The model wind turbine is with $0.894 \mathrm{~m}$ rotor diameter and 0.89 hub height.

The turbine rotates counterclockwise (CCW) with a tip speed ratio $(\mathrm{TSR})=6.13 \%$ is the blockage ratio, which is the ratio between rotor's swept area to the cross-section area of the tunnel. Its three blades, milled from aluminum, were designed using NREL S826 airfoil overall the blade i.e. from the root to the tip.

\section{RESULTS AND DISCUSSION}

\subsection{Power output}

The values of power and thrust coefficients are listed on Table 1. Equations (22) and (23) show that Power coefficient is the ratio of power extracted by the turbine to the available power in freestream air, and thrust coefficient is the ratio between axial force on the rotor and the dynamic force of the wind.

$$
\begin{aligned}
C_{p} & =\frac{P}{0.5 \rho U_{\text {ref }}^{3} A} \\
C_{T} & =\frac{T}{0.5 \rho U_{\text {ref }}^{2} A}
\end{aligned}
$$

Where $P$ is the extracted Power by the turbine, $T$ is the force acting on the rotor in downstream direction, $\mathrm{A}$ is the swept area of the rotor $\left(A=\pi D^{2} / 4\right), \rho$ is the air density and $U_{\text {ref }}$ is the freestream velocity.

Table 1. Power and thrust coefficients compared to experimental data.

\begin{tabular}{|l|c|l|l|l|}
\hline Model/coefficient & $C_{p}$ & Error\% & $C_{\mathrm{t}}$ & Error\% \\
\hline Standard k- $\varepsilon$ & 0.282 & -39.7 & 0.81 & -7 \\
\hline Realizable k- $\varepsilon$ & 0.412 & -11.88 & 0.833 & -4.3 \\
\hline SST k- $\omega$ & 0.403 & -13.7 & 0.787 & -9.53 \\
\hline K-kl- $\omega$ transition & 0.424 & -9.14 & 0.83 & -4.5 \\
\hline Transition SST & 0.414 & -11.28 & 0.805 & -7.5 \\
\hline experiment & 0.467 & & 0.87 & \\
\hline
\end{tabular}

A modest underestimation of power coefficient by most of the models is noticed with the least error for the K-kl- $\omega$ transition model. The Standard k- $\varepsilon$ model shows high deviation (unaccepted) which is returned to its poor quality near wall regions. In another study, which is operated in the time of writing this paper, error percentage reduced to about $7 \%$ compared to $13.7 \%$ considering unsteady behavior with SST k- $\omega$ model. this indicates to the influence of the steady assumption on the power prediction. Simulations show reasonable prediction for the thrust coefficients.

Transition models reveal more accurate estimations compared to fully developed models due to its capability of detecting separation phenomena specially at the range of moderate Reynolds number as in our case $(\operatorname{Re}=105)$. This conclusion agrees with [25]; in which, the Transition SST was compared with fully turbulent model to predict the performance of a VAWT.

\subsection{Velocity profile}

Axial velocity is plotted versus non-dimensional distance $\mathrm{z} / \mathrm{R}$ in Figure 4, where $\mathrm{R}$ is the rotor radius. For validation, the only data available from the experiments are at $6 \mathrm{D}$ behind the turbine, where $\mathrm{D}$ is the diameter of the turbine in meters.

Velocity profile turns from semi $\mathrm{W}$ shape at $3 \mathrm{D}$ to a gaussian profile at $6 \mathrm{D}$. Except for the K-kl- $\omega$ transition model, models show quite close predictions at $\mathrm{x} / \mathrm{D}=3$ (near region) in contrast

to significant variance between models at $\mathrm{x} / \mathrm{D}=6$ (far region). For SST k- $\omega$ and standard k- $\epsilon$ models, results are reasonable; otherwise, K-kl- $\omega$ transition model show higher velocities at both 3D and 6D. Velocity curve for transition SST model at 3D fits well with SST k- $\omega$ model; however, it has higher velocities at $6 \mathrm{D}$.

Wake width is defined as the region in which velocity is lower than freestream velocity [26] i.e. wake extends between the points at which velocity $\mathrm{u}=10 \mathrm{~m} / \mathrm{s}$. At $3 \mathrm{D}$, Wake nearly extends radially from $\mathrm{z} / \mathrm{R}=-1.1$ to +1.1 for most models. At

$6 \mathrm{D}$, width nearly expands from to $\mathrm{z} / \mathrm{R}=-1.2$ to +1.4 compared to $\mathrm{z} / \mathrm{R}=-1.5$ to 1.65 for experimental data. Simulations show narrower wake region which means that it is sensitive to tunnel blockage and requires a finer meshing near the walls of the tunnel which will rise the computational time significantly. Figure 5 illustrates the contours of axial velocity at different distances downstream ( $\mathrm{x} / \mathrm{D}=1,3$, and 6). Contours depict the wake recovery as directing downstream. All models show misrepresentation of the proper rounded shape of velocity distribution as shown from experimental data, this is returned to the steady assumption; thereby, it will be a deficiency or an error source in case of simulating tandem turbines. Also,

contours confirm that both transitional models overestimate the velocity at the far region, and the fully turbulent models resembles in their behavior

\subsection{Turbulence intensity}

Turbulence level is represented by equation Error! Reference source not found.; where, TI is turbulence intensity, $v^{\prime}$ is the root-mean-square of turbulence fluctuations, and $U$ is mean velocity. Turbulence intensity is a vital parameter to be considered in farms layout and turbine design processes as it is the main source of fatigue loads on the turbines [27]. As depicted in Figure 6, except for the k-kl- $\omega$ transition model, all models failed to predict turbulence level. High TI levels of the $\mathrm{k}-\mathrm{kl}-\omega$ transition model are corresponding to overestimated velocity values at the wake region. In a LES study [7], turbulence intensity at near wake region is higher than inflow turbulence intensity due to interaction with the turbine; After that, it decays at the far region. This is not the case for our simulations; at which, turbulence intensity is lower than inflow 
TI through the whole wake region. Maximum values of TI are at the tip of the blades accompanying high vorticity and mixing rate.

$T I=\frac{v^{\prime}}{U}$ and $v^{\prime}=\sqrt{\left.\frac{1}{3} \overline{\left(u^{\prime 2}\right.}+\overline{v^{\prime 2}}+\overline{w^{\prime 2}}\right)}$

\subsection{Turbulent kinetic energy}

Turbulent kinetic energy is calculated from equation (25). From Figure 7, Two peaks of TKE are observed behind the tip of the turbine and lower values behind the hub. These peaks coincide with high turbulence intensity and high shear due to high mixing between low velocity region and surrounding high velocities. TKE Slightly decreases for all models from 3D to $6 \mathrm{D}$ except the k-kl- $\omega$ transition model for which peaks values significantly decreases.

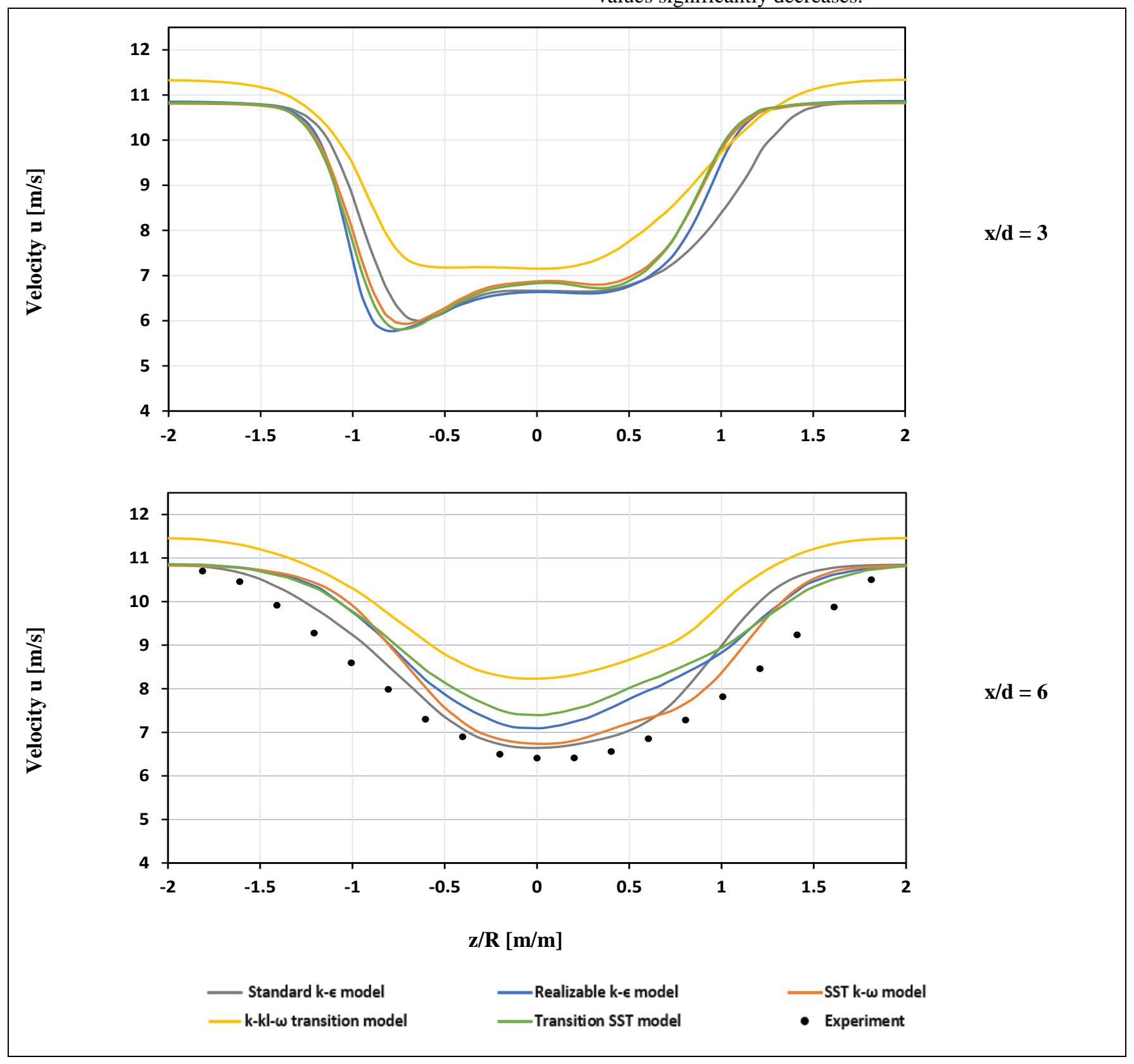

Figure 4 . The axial velocity profile at a vertical line at the hub height, top: at $\mathrm{x} / \mathrm{d}=3$ behind the turbine and bottom: at $\mathrm{x} / \mathrm{d}=6$. 


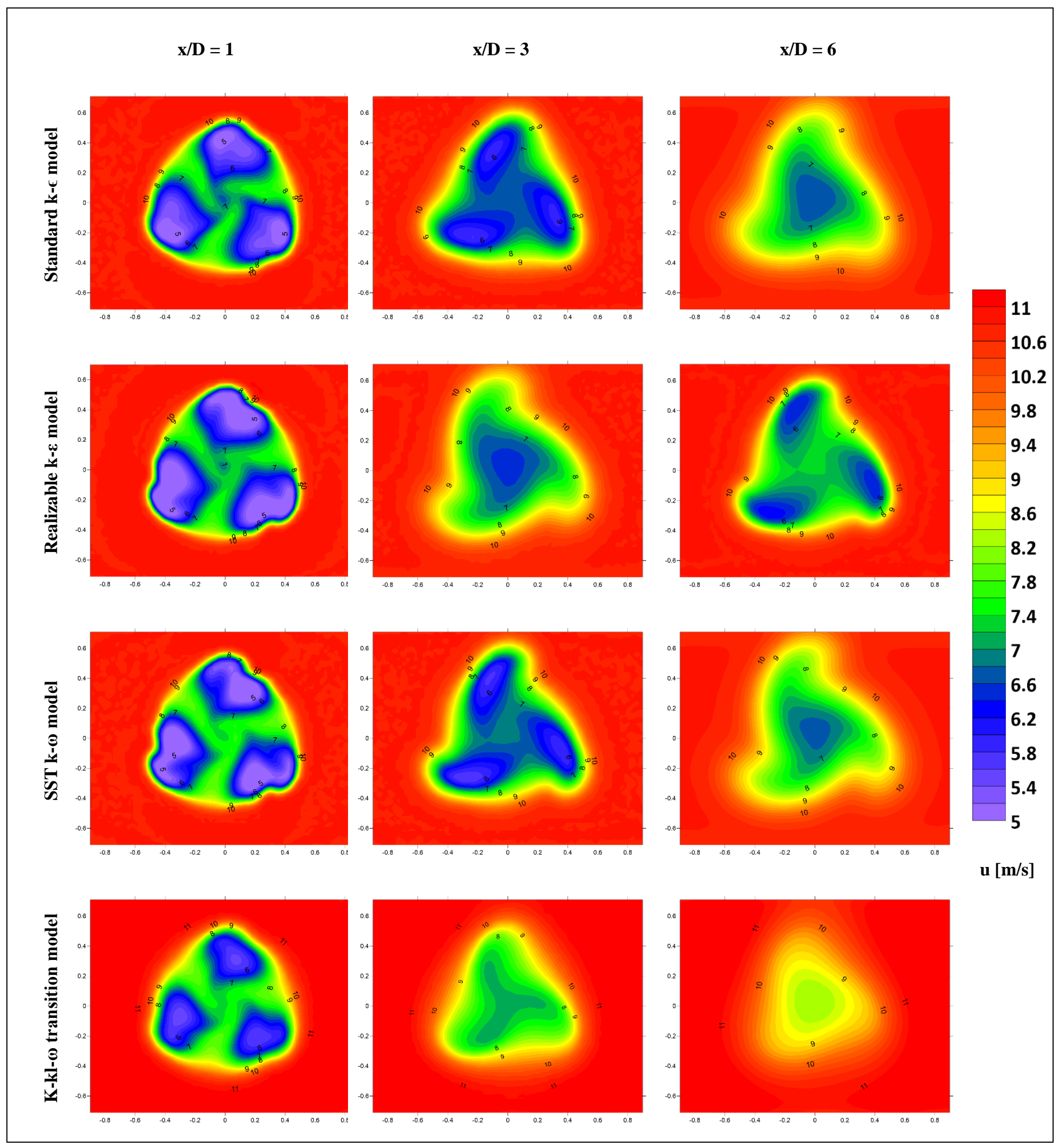



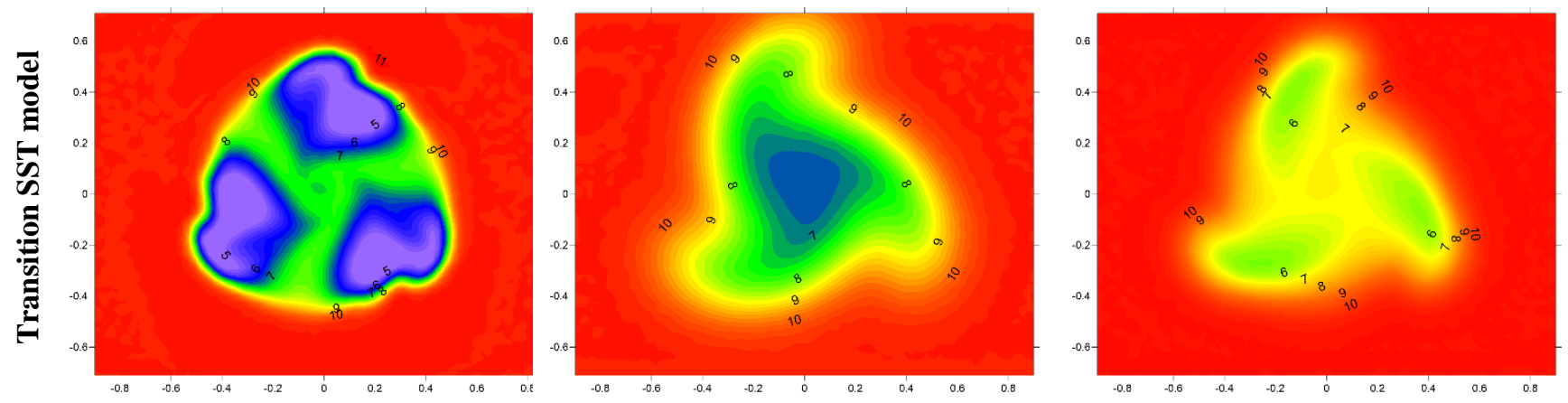

昰

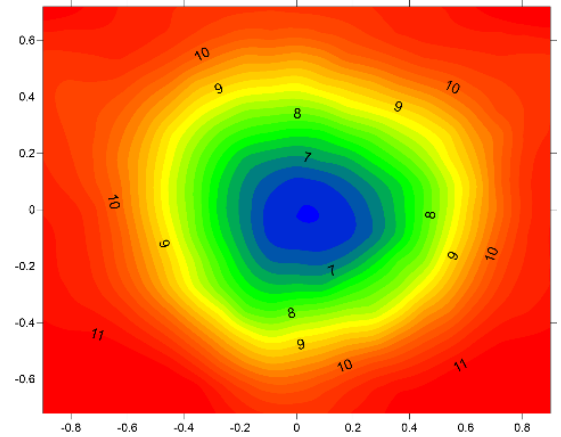

Figure 5. Axial velocity (u) contours at $\mathrm{x} / \mathrm{D}=1,3$, and 6

\subsection{Turbulent kinetic energy}

Turbulent kinetic energy is calculated from equation (25). From Figure 7, Two peaks of TKE are observed behind the tip of the turbine and lower values behind the hub. These peaks coincide with high turbulence intensity and high shear due to high mixing between low velocity region and surrounding high velocities. TKE Slightly decreases for all models from 3D to $6 \mathrm{D}$ except the k-kl- $\omega$ transition model for which peaks values significantly decreases. At $\mathrm{x} / \mathrm{D}=6$, simulations are compared to three different profiles of experimental data which is based on a two-component LDA. As formulated in equations (26), (27), and (28), TKE1 considers the two components $\overline{u^{\prime 2}}$ and $\overline{v^{\prime 2}}$, TKE2 assumes isotropic normal stresses approximation i.e. $\overline{u^{\prime 2}}=\overline{v^{\prime 2}}=\overline{{w^{\prime 2}}^{2}}$; finally, TKE3 assumes $\overline{{v^{\prime}}^{2}}=\overline{{w^{\prime}}^{2}}$.

$$
\begin{aligned}
& T K E=\frac{1}{2}\left(\overline{u^{\prime 2}}+\overline{v^{\prime 2}}+\overline{w^{\prime 2}}\right) \\
& T K E 1=\frac{1}{2}\left(\overline{u^{\prime 2}}+\overline{v^{\prime 2}}\right) \\
& T K E 2=\frac{3}{2}\left(\overline{u^{\prime 2}}\right) \\
& T K E 3=\frac{1}{2}\left(\overline{u^{\prime 2}}+2 * \overline{v^{\prime 2}}\right)
\end{aligned}
$$

Where $\overline{u^{\prime 2}}, \overline{v^{\prime 2}}$ and $\overline{w^{\prime 2}}$ are the fluctuating velocity components.

returned the incorrect evaluation of TI and TKE to the obvious anisotropy of turbines wake contrary to isotropic assumption adopted in eddy-viscosity based models. Comparing simulations with TKE2, which assumes isotropic stresses values

depending on measured velocities, illuminate the idea of that it is not only an isotropy issue; furthermore, it is a too much turbulence damping issue. Hence, used turbulence generation and dissipation terms need to be reformulated and reassessed with wind turbine applications.

Like velocity contours, TKE contours at Figure 8 misdiagnose the rounded shape; however, they depict the decay of TKE as we go far from the turbine and that the maximum values are forming a ring behind the blade tip. 
Vol. 2, 2020

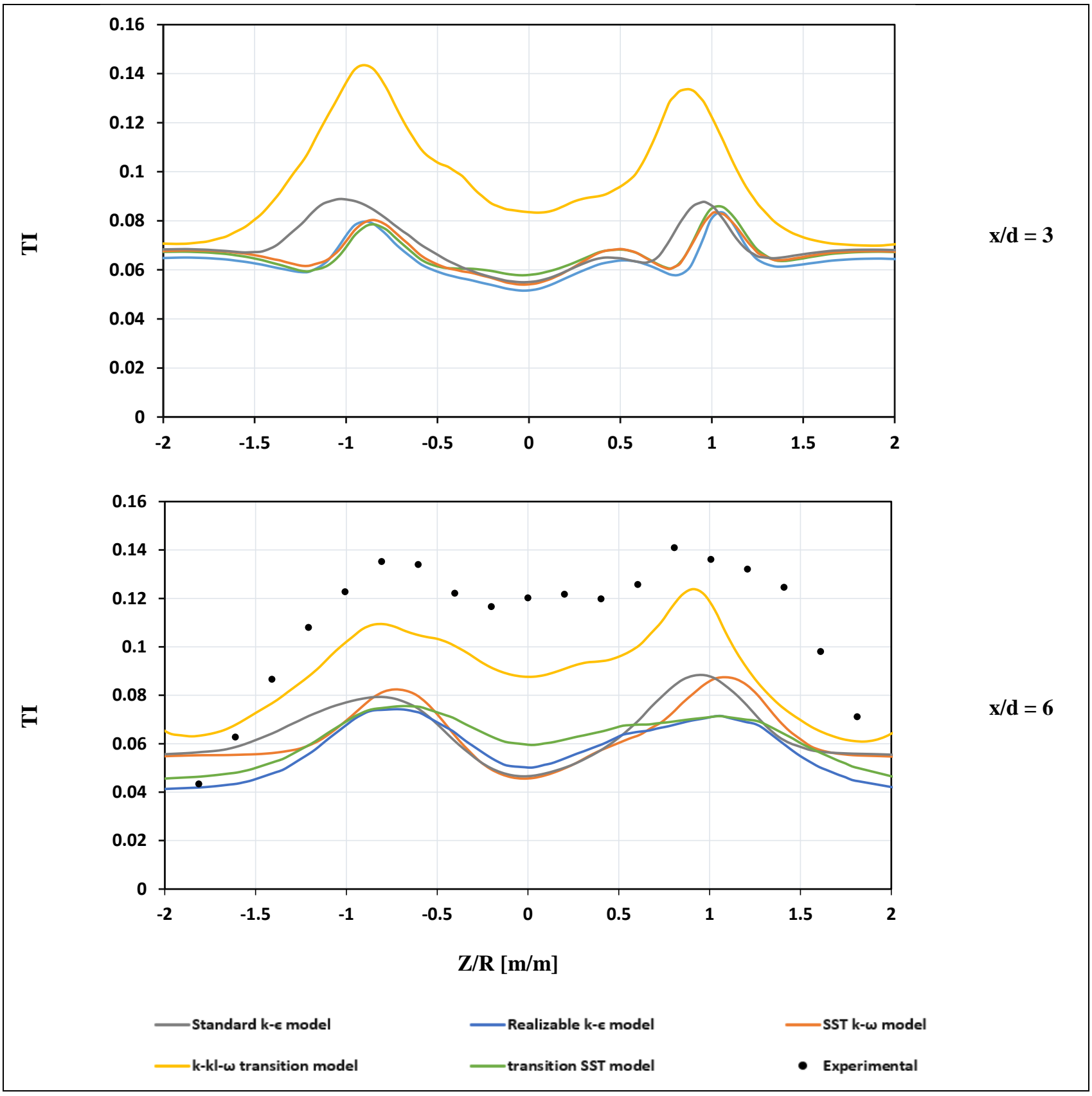

Figure 6 . The turbulence intensity profile at a vertical line at the hub height, top: at $\mathrm{x} / \mathrm{D}=3$ behind the turbine and bottom: at $\mathrm{x} / \mathrm{D}=6$. 


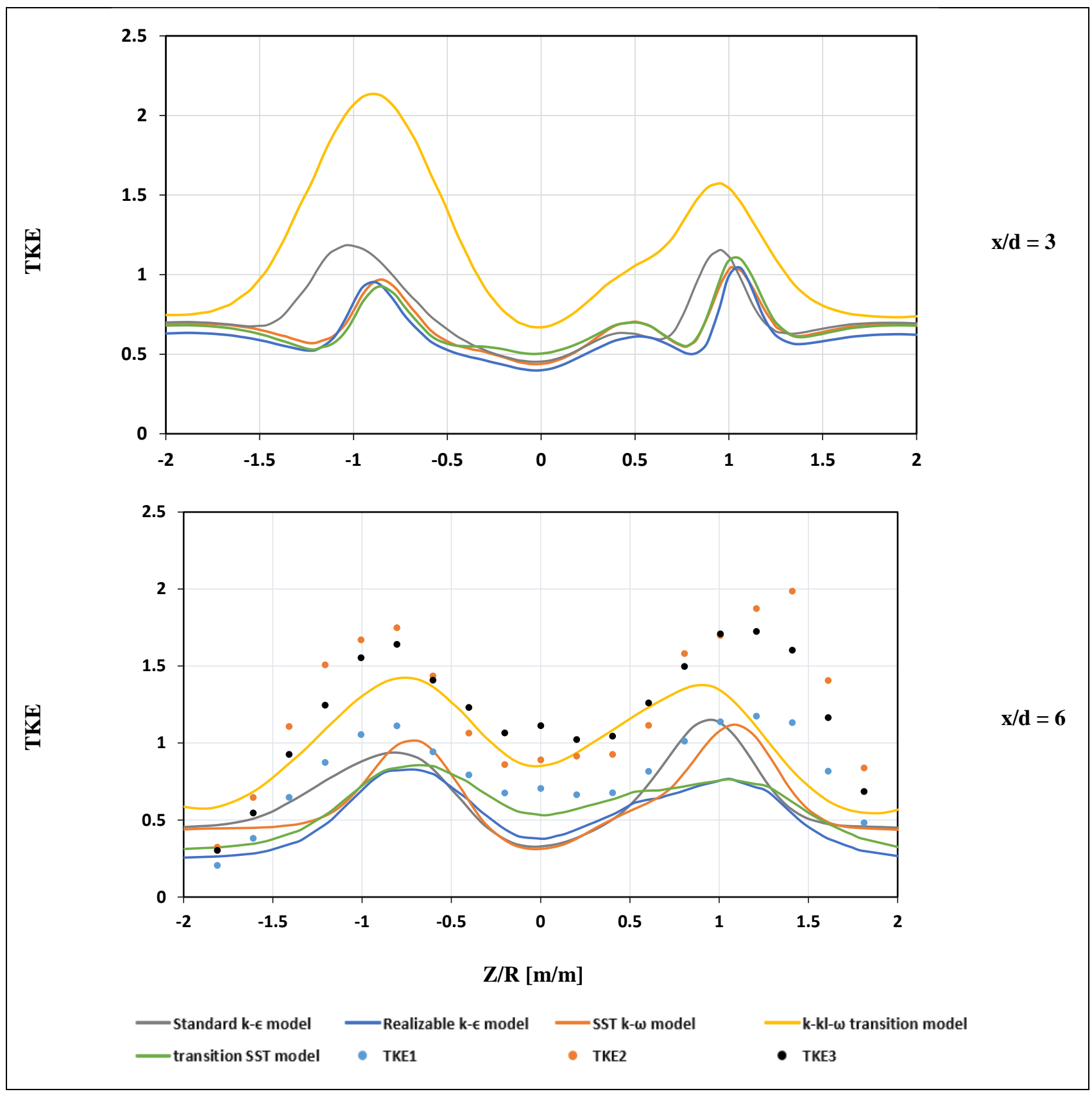

Figure 7. Turbulent kinetic energy $\left(\mathrm{m}^{2} / \mathrm{s}^{2}\right)$ at a horizontal line at the level of hub height at $\mathrm{x} / \mathrm{D}=3$ and 6 .

\section{CONCLUSION}

A Comparison between five turbulence models was carried out to investigate the potential of these models of predicting wake flow characteristics behind the model wind turbine. The study was operating at a uniform high turbulence intensity inflow condition. Turbulence modelling was based on Steady Reynolds average Navier Stokes (RANS) with Multiple reference frame method. Rotor was modeled, but nacelle and tower were excluded to reduce the computational cost. Results of the simulations can be remarked in the next points.

- A slight underestimation of power coefficient for the models expect Standard k- $\varepsilon$ model which shows unacceptable result due to its poor quality for wall interaction cases.

- Transition models are more accurate than fully developed models in predicting power and thrust coefficients due to its capability of detecting separation phenomena on the blades. 

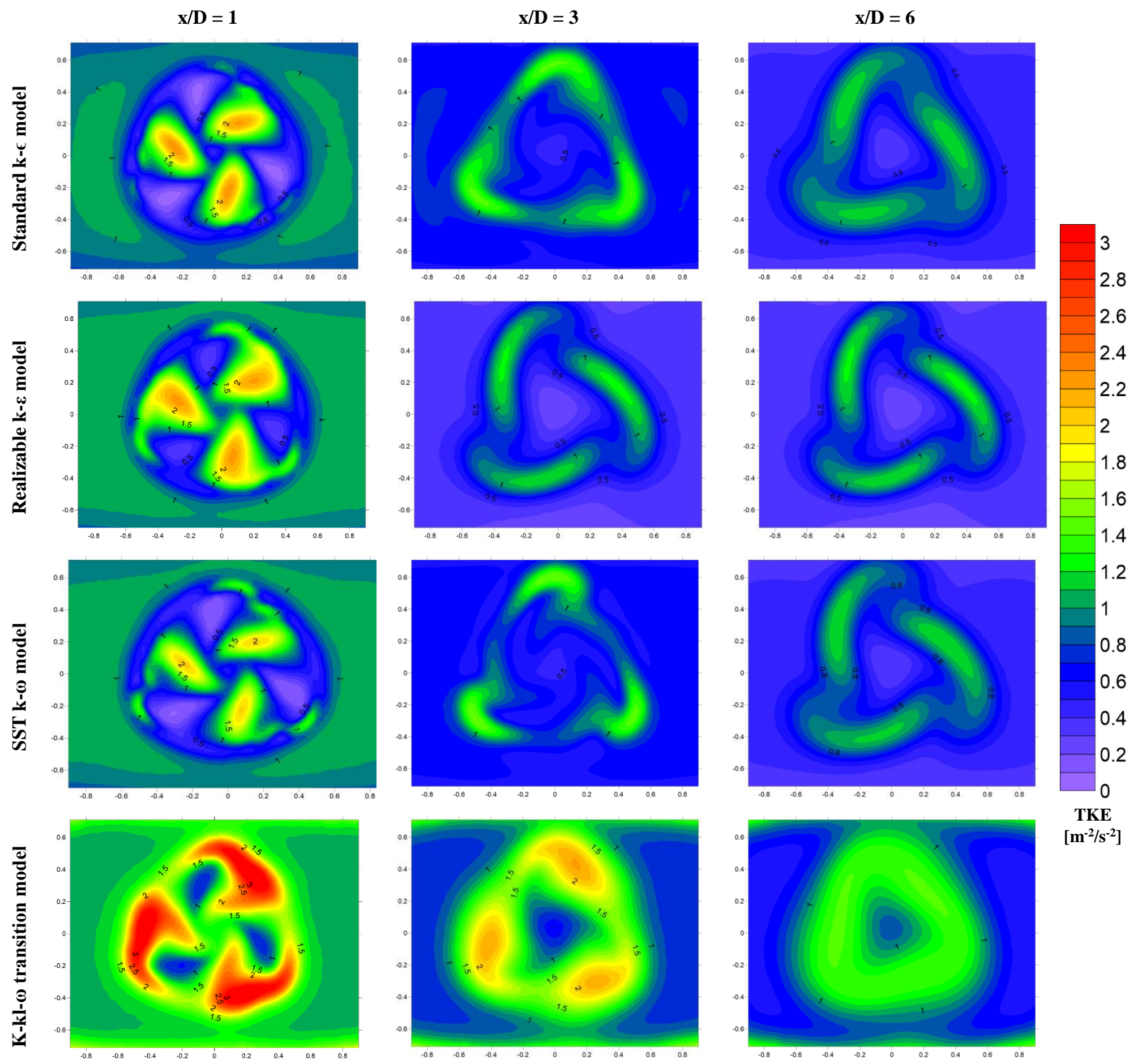

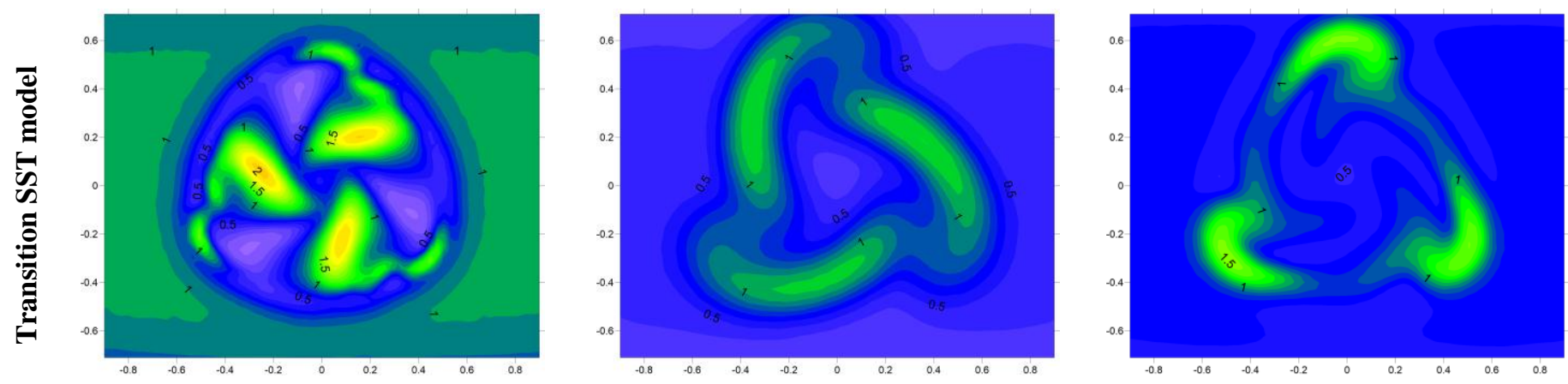

닐

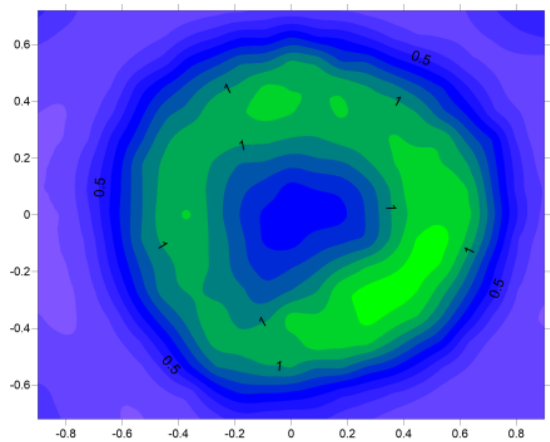

놀

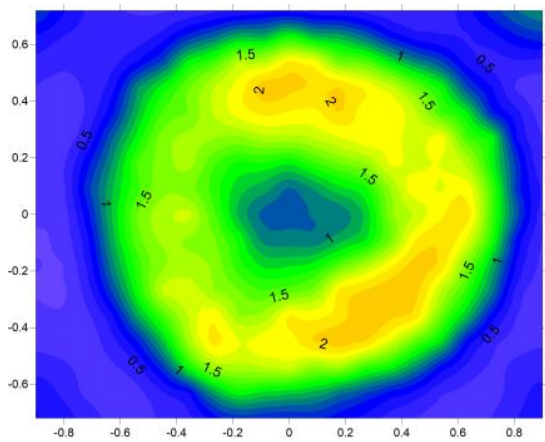

告

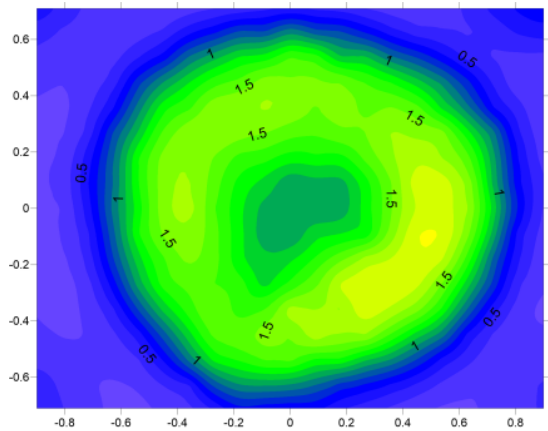

Figure 8. Turbulent kinetic energy (TKE) contours at $\mathrm{x} / \mathrm{D}=1,3$, and 6

- Fully developed models predict velocity profile at $\mathrm{x} / \mathrm{D}$ more accurately than transitional ones.

- Simulations show narrower wake region which means higher mixing between wake region and deficit velocity region and higher sensitivity to tunnel blockage.
- All models show a disability to depict the rounded shape of the velocity contours, this is returned to steady behavior assumption

- Both of Turbulence intensity and turbulent kinetic energy are underpredicted by all models. This deviation is resulted from isotropic assumption of RANS plus overdamping of turbulence. 
Upcoming studies will include studying unsteady behavior at different operating conditions and assess transitional models at low turbulence intensity values.

\section{NOMENCLATURE}

\begin{tabular}{|l|l|}
\hline CCW & Counterclockwise \\
\hline CFD & Computational Fluid Dynamics \\
\hline D & Rotor Diameter \\
\hline h & Hub height \\
\hline HAWT & Horizontal axis wind turbine \\
\hline LES & Laser doppler anemometer \\
\hline MRF & Multi reference frame \\
\hline NTNU & $\begin{array}{l}\text { Norwegian University of Sciences and } \\
\text { Technology }\end{array}$ \\
\hline R & Rotor radius \\
\hline Re & Reynolds number \\
\hline RSM & Reynolds stress model \\
\hline TI & Turbulence intensity \\
\hline TKE & Turbulent kinetic energy \\
\hline TSR & Tip Speed Ratio \\
\hline VAWT & Vertical axis wind turbine \\
\hline VR & Viscosity Ratio \\
\hline
\end{tabular}

Refrences

1. Global Wind Energy Council, Global Wind Report 2019. 2019.

2. Gaumond, M., et al., Evaluation of the wind direction uncertainty and its impact on wake modeling at the Horns Rev offshore wind farm. Wind Energy, 2014. 17(8): p. 1169-1178.

3. Choudhry, A., et al., Effects of wake interaction on downstream wind turbines. Wind Engineering, 2014. 38(5): p. 535-547.

4. Lundquist, J.K., et al., Costs and consequences of wind turbine wake effects arising from uncoordinated wind energy development. Nature Energy, 2019. 4(1): p. 2634.

5. Mehta, D., et al., Large Eddy Simulation of wind farm aerodynamics: A review. Journal of Wind Engineering and Industrial Aerodynamics, 2014. 133: p. 1-17.

6. Sedaghatizadeh, N., et al., Modelling of wind turbine wake using large eddy simulation. Renewable Energy, 2018. 115: p. 1166-1176.

7. Mo, J.-O., et al., Large eddy simulation of the wind turbine wake characteristics in the numerical wind tunnel model. Journal of Wind Engineering and Industrial Aerodynamics, 2013. 112: p. 11-24.

8. Fleming, P., et al., Simulation comparison of wake mitigation control strategies for a two-turbine case. Wind Energy, 2015. 18(12): p. 2135-2143.

9. Vollmer, L., et al., Estimating the wake deflection downstream of a wind turbine in different atmospheric stabilities: an LES study. Wind Energ. Sci, 2016. 1: p. 129-141.

10. Cabezón, D., E. Migoya, and A. Crespo, Comparison of turbulence models for the computational fluid dynamics simulation of wind turbine wakes in the atmospheric boundary layer. Wind Energy, 2011. 14(7): p. 909-921.

11. van der Laan, P.M., et al. Nonlinear eddy viscosity models applied to wind turbine wakes. in International Conference on aerodynamics of Offshore Wind Energy Systems and wakes (ICOWES 2013). 2013. Technical University of Denmark.

12. El Kasmi, A. and C. Masson, An extended $k-\varepsilon$ model for turbulent flow through horizontal-axis wind turbines. Journal of Wind Engineering and Industrial Aerodynamics, 2008. 96(1): p. 103-122.

13. van der Laan, M. and S. Andersen. The turbulence scales of a wind turbine wake: A revisit of extended k-epsilon models. in J. Phys.: Conf. Ser. 2018.

14. van der Laan, M.P., et al., An improved $k-\epsilon$ model applied to a wind turbine wake in atmospheric turbulence. Wind Energy, 2015. 18(5): p. 889-907.

15. Imiela, M. CFD Simulations of theNew MEXICORotor Experiment under Yawed Flow. in Journal of Physics: Conference Series. 2018. Institute of Physics (IOP) Publishing.

16. Schottler, J., Bartl, Jan, \& Mühle, Franz., Wind tunnel experiments on wind turbine wakes in yaw [Data set]. 2018.

17. ANSYS Inc, ANSYS Fluent Theory Guide. 2013.

18. Versteeg, H.K. and W. Malalasekera, An introduction to computational fluid dynamics: the finite volume method. 2007: Pearson education.

19. Shih, T.-H., et al., A new $k$ - $\epsilon$ eddy viscosity model for high reynolds number turbulent flows. Computers \& Fluids, 1995. 24(3): p. 227-238.

20. Menter, F.R., Influence of freestream values on k-omega turbulence model predictions. AIAA Journal, 1992. 30(6): p. 1657-1659.

21. Menter, F.R., Two-equation eddy-viscosity turbulence models for engineering applications. AIAA journal, 1994. 32(8): p. 1598-1605.

22. Menter, F.R., Eddy viscosity transport equations and their relation to the $k-\varepsilon$ model. 1997.

23. Menter, F.R., M. Kuntz, and R. Langtry, Ten years of industrial experience with the SST turbulence model. Turbulence, heat and mass transfer, 2003. 4(1): p. 625632.

24. Walters, D.K. and D. Cokljat, A three-equation eddyviscosity model for Reynolds-averaged Navier-Stokes 
Vol. 2, 2020

simulations of transitional flow. Journal of fluids engineering, 2008. 130(12).

25. Lanzafame, R., S. Mauro, and M. Messina, $2 D C F D$ Modeling of H-Darrieus Wind Turbines Using a Transition Turbulence Model. Energy Procedia, 2014. 45: p. 131-140.

26. Mo, J.-O., et al., Effects of wind speed changes on wake instability of a wind turbine in a virtual wind tunnel
Journal of Engineering Research (ERJ)

using large eddy simulation. Journal of Wind Engineering and Industrial Aerodynamics, 2013. 117: p. 38-56.

27. Ismaiel, A. and S. Yoshida, Study of Turbulence Intensity Effect on the Fatigue Lifetime of Wind Turbines. Evergreen, 2018. 05: p. 25-32. 